\title{
Voyance, chronotopie et intertextualité dans Dora Bruder de Patrick Modiano
}

\author{
Helge Vidar Holm (Université de Bergen, Norvège)
}

\begin{abstract}
In Dora Bruder, Patrick Modiano gives the following comment on his own novelistic writing: "Like many writers before me, I believe in coincidence, and, sometimes, in the novelist's gift for clairvoyance - the word 'gift' not being the right one, for it implies a kind of superiority. Clairvoyance is simply part of the profession: the essential leaps of imagination, the need to fix one's mind on detail - to the point of obsession, in fact - so as not to lose the thread and give in to one's natural laziness. All this tension, this cerebral exercise, may well lead in the long run to 'flashes of intuition concerning events past and future', as defined by Larousse dictionary under 'clairvoyance'." (Dora Bruder (Paris 1997), English edition: The Search Warrant, Harvill Secker, London 2014, p. 47-48).
\end{abstract}

By analyzing some selected passages from this novel, I study some relations between M. Bakhtin's theory of the chronotope and the effects of the novelist Modiano's "gift for clairvoyance" as presented in the quotation above. I also comment on some representations of intertextuality and intratextuality in Dora Bruder.

Keywords: Patrick Modiano, Dora Bruder, chronotope, intertextuality, clairvoyance

Prenons comme point de départ la citation suivante du roman de 1997 de Patrick Modiano, Dora Bruder :

Comme beaucoup d'autres avant moi, je crois aux coïncidences et quelquefois à un don de voyance chez les romanciers - le mot 'don' n'étant pas le terme exact, parce qu'il suggère une sorte de supériorité. Non, cela fait simplement partie du métier: les efforts d'imagination, nécessaires à ce métier, le besoin de fixer son esprit sur des points de détail - et cela de manière obsessionnelle - pour ne pas perdre le fil et se laisser à aller à sa paresse -, toute cette tension, cette gymnastique cérébrale peut sans doute provoquer à la longue de brèves intuitions « concernant des événements passés ou futurs », comme l'écrit le dictionnaire Larousse à la rubrique « Voyance » ${ }^{1}$ (Dora Bruder, éd. Folio, p.5253).

En m'appuyant sur la théorie des chronotopes, développée par le poéticien russe Mikhaïl Bakhtine, j'étudierai la correspondance entre cette théorie bakhtinienne et les effets et les représentations du phénomène de voyance, tels que conçus par Modiano et exprimés dans

\footnotetext{
${ }^{1}$ Dora Bruder, pp. 52-53.
} 
son roman Dora Bruder. De plus, j'analyserai quelques effets d'intertextualité et d'intratextualité ${ }^{2}$ dans ce texte romanesque.

Bakhtine introduisait et développait le concept littéraire de chronotope dans un essai de poétique historique intitulé en français « Formes et temps du chronotope dans le roman », rédigé principalement en 1937-1938, avec un chapitre d'observations finales rajoutés en conclusion en 1973. Voilà comment il présente le terme au début de son essai :

Nous appellerons chronotope, ce qui se traduit, littéralement, par «temps-espace » : la corrélation essentielle des rapports spatio-temporels, telle qu'elle a été assimilée par la littérature. Ce terme est propre aux mathématiques; il a été introduit et adapté sur la base de la théorie de la relativité d'Einstein. Mais le sens spécial qu'il y a reçu nous importe peu. Nous comptons l'introduire dans l'histoire littéraire presque (mais pas absolument) comme une métaphore. Ce qui compte pour nous, c'est qu'il exprime l'indissolubilité de l'espace et du temps (celui-ci comme quatrième dimension de l'espace). Nous entendons chronotope comme une catégorie littéraire de la forme et du contenu, sans toucher à son rôle dans d'autres sphères de la culture ${ }^{3}$.

La référence de base est à trouver chez Kant, qui dans son Esthétique Transcendentale définit l'espace et le temps comme les conditions a priori de notre perception du monde. Bakhtine se déclare en principe d'accord avec Kant en ce qui concerne la signification du temps et de l'espace pour le processus de la connaissance, tout en précisant que c'est le rôle du chronotope dans l'art littéraire, notamment dans les spécificités du genre romanesque, qu'il est en train de d'élaborer.

Le chronotope appartient donc à l'art du roman. Dans notre contexte, on pourrait dire qu'il appartient à l'art du romancier, si nous nous appuyons sur la pratique de Modiano, à la fois littéraire pure et métalittéraire, dans le roman Dora Bruder. Pour la pratique métalittéraire, je pense notamment aux commentaires de l'auteur, p.ex. celui donnée dans la citation liminaire du présent article.

Une partie centrale de la critique émise contre l'emploi dans l'analyse littéraire du concept du chronotope, a trait à son rapport à la narratologie, notamment en ce qui concerne les dimensions temporelles du texte littéraire ${ }^{4}$. Dans la narratologie, on fait la distinction entre le temps du récit (temps de la narration) et le temps de l'histoire (temps raconté), et à partir de discussions diverses de cette dualité fondamentale de tout texte narratif, nous pouvons analyser différents aspects de l'ordre, de la durée, de la fréquence, du mode et de la voix du récit, pour citer les termes employés par Gérard Genette dans son essai de 1972 qui fait date dans l'histoire de la narratologie: «Discours du récit ${ }^{5}$. La question qu'on pourrait se poser serait donc celle-ci : les implications de l'emploi du concept du chronotope dans l'analyse littéraire, sont-elles de nature différentes de celles contenues dans l'analyse narratologique ? $\mathrm{Ou}$, en d'autres mots, le concept du chronotope donne-t-il la clef à de nouvelles portes d'accès à l'oeuvre littéraire ou nous aide-t-il plutôt à enfoncer des portes plus ou moins grand ouvertes déjà depuis un certain temps ?

Dans notre cas, où nous mettons la chronotopie en relation avec la notion de voyance du romancier, telle que développée par Modiano, l'approche chronotopique implique une

\footnotetext{
${ }^{2}$ Une intertextualité entre deux oeuvres du même auteur.

${ }^{3}$ Bakhtine 1978, p. 237

${ }^{4}$ Voir p. ex. Greve \& Gaasland 2004, notamment pp. 39-40.

${ }^{5}$ Genette 1972, pp. 65-267.
} 
compréhension du rôle du temps et du lieux (chronos et topos) nettement différente de celle rendue possible par l'approche narratologique. Pourtant les deux approches peuvent bien se compléter. Nous allons le voir notamment dans une analyse de l'avant-dernier paragraphe du roman, mais d'abord, commençons par le début, par l'incipit de Dora Bruder :

Il y a huit ans, dans un vieux journal, Paris-Soir, qui datait du 31 décembre 1941, je suis tombé à la page trois sur une rubrique: « D'hier à aujourd'hui ». Au bas de celle-ci, j'ai lu:

«PARIS

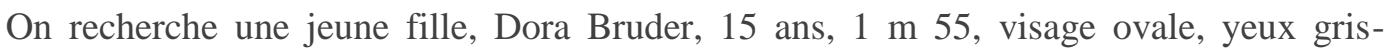
marron, manteau sport gris, pull-over bordeaux, jupe et chapeau bleu marine, chaussures sport marron. Adresser toutes indications à M. et Mme Bruder, 41 boulevard Ornano, Paris. »

Le rapport du narrateur aux lieux précis mentionnés dans le texte romanesque est situé à la fois dans le temps du récit et dans le temps de l'histoire. Ces lieux précis sont en effet des clefs pour une compréhension de l'effet de la théorie de la voyance de romancier, présentée dans notre citation liminaire. Nous allons le voir en détail vers la fin du présent article.

Passons maintenant à la suite immédiate dans le roman du commentaire métalittéraire dans ma première citation. C'est toujours le narrateur homodiégétique ${ }^{6}$ qui parle de sa propre situation professionnelle:

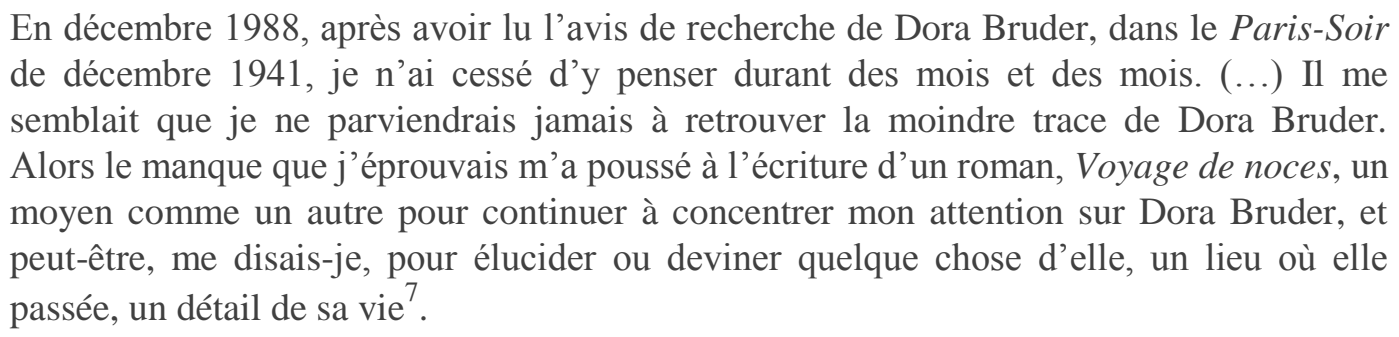

En fait, Voyage de noces, de 1990, est un roman à la fois tout autre que Dora Bruder et, thématiquement, très proche de ce dernier. Pour le romancier Modiano, la recherche factuelle n'est qu'une partie des moyens qu'il faut mettre en œuvre dans la préparation de la rédaction d'un roman. Le plus important, c'est de mettre en oeuvre son don de voyance, et donc les efforts d'imagination, nécessaires à ce métier, pour le citer encore une fois. Cela peut passer par l'écriture d'un roman tout différent au niveau de l'action et la vie des personnages, comparé à ce que sera plus tard le roman Dora Bruder. En effet, Voyage de noces est construit sur une quête d'identité, donc sur un thème majeur présent dans toute l'œuvre de Patrick Modiano. Et sans aucun doute, ce roman est étroitement lié à Dora Bruder. Entre autre, un des personnages principaux, Ingrid Teyrsen, est en partie clairement inspirée de la jeune juive Dora.

Ce qui à mon avis est particulièrement intéressant pour notre analyse, c'est de voir comment Modiano décrit son propre chemin vers la rédaction de son roman Dora Bruder, où d'ailleurs je dirai que le personnage principal n'est point la jeune fille Dora, mais plutôt le narrateur du texte. Donc, le narrateur homodiégétique peut en effet en cacher un autre, un

\footnotetext{
${ }^{6}$ Ou plus spécifiquement autodiégétique ? Voir infra.

${ }^{7}$ Dora Bruder, pp. 52-53. C'est moi qui souligne.
} 
narrateur autodiégétique ${ }^{8}$. Voilà un aspect narratologique avec grande importance pour l'appartenance générique et, partant, pour la compréhension de l'oeuvre. En effet, si l'action principal est constituée par la recherche de l'histoire de la vie vécue par Dora, ce roman est aussi celui d'une quête d'identité, où l'histoire de l'enfance et de la famille de l'auteur joue un rôle essentiel ${ }^{9}$.

Dans Dora Bruder, le rapport du narrateur aux lieux précis mentionnés dans ce texte romanesque se trouve fréquemment à la fois dans le temps du récit et dans le temps de l'histoire. Un exemple serait un passage où Modiano, alias le narrateur du roman Dora Bruder, dit ceci, à propos de la rédaction de Voyage de noces : «Je me rends compte aujourd'hui qu'il m'a fallu écrire deux cent pages pour capter, inconsciemment, un vague reflet de la réalité [...] le seul moment du livre où, sans le savoir, je me suis approché d'elle, ${ }^{10}$ dans l'espace et le temps ${ }^{11}$. »

Ce moment de Voyage de noces, c'est un passage où deux des personnages principaux, Rigaud et Ingrid, sortent du métro un jour d'hiver et de neige, près du Pensionnat Saint-Cœurde-Marie « $[. .$.$] d'où Dora Bruder devait faire une fugue, un soir de décembre et au cours$ duquel la neige était peut-être tombée sur Paris. $»^{12}$

Nous voyons ici combien les éléments spatiaux et temporels sont importants pour la recherche effectuée par le narrateur. A l'époque où il rédigeait Voyage de noces, Modiano ignorait, selon ses propres mots, nous l'avons vu, toutes des circonstances de la fugue de Dora. Cependant, en écrivant un roman sur une situation et un destin similaires à ceux qu'il pouvait imaginer être ceux de Dora, il est arrivé à s'approcher d'elle, dans l'espace et le temps.

En effet, dans les année 1990, Modiano a pu obtenir, par son propre travail d'investigateur aussi bien que par l'intermédiaire de l'historien et avocat Serge Klarsfeld, beaucoup de détails sur l'existence de la jeune juive Dora Bruder ${ }^{13}$. Parmi ces détails, ceux concernant le pensionnat Saint-Cœur-de-Marie jouent un rôle particulièrement important dans la rédaction du roman, tel que nous allons le voir infra.

Le lien entre la théorie modianienne de « voyance » et l'importance du chronotope, explique selon moi, avec ses implications intratextuelles, ce passage un peu énigmatique, donnée dans mon avant-dernière citation: « le seul moment du livre où, sans le savoir, je me suis approché d'elle, dans l'espace et le temps. » Il y a une forme de chronotope, doublement bakhtinien, dans ce roman. On pourrait l'appeler le « chronotope intertextuel », en combinant deux concepts d'origine bakhtinienne. Doublement bakhtinien donc, puisque Julia Kristeva lança son concept d'intertextualité à partir d'une discussion qu'elle présenta du concept fondamental chez Bakhtine de dialogisme en 1967, dans l'article «Bakhtine, le mot, le dialogue et le roman $\gg 14$.

En plus de l'usage intratextuel de Voyage de noces, il y a dans Dora Bruder des jeux intertextuels et chronotopiques par des références directes à deux œuvres majeures de la

\footnotetext{
${ }^{8}$ Voir Genette op. cit., p. 253

${ }^{9}$ Pour ma part, je me range donc du côté de ceux qui regardent cette œuvre comme un roman, et non comme une biographie ou un texte principalement documentaire. Pour un autre avis que le mien à ce propos, voir par exemple Bem 2000.

${ }^{10}$ d'elle = de Dora

${ }^{11}$ Dora Bruder, pp. 53-54. C'est moi qui souligne.

${ }^{12}$ Loc.cit.

${ }^{13}$ A ce propos, voir p. ex. Hilsum 2012 et Holm 2017.

${ }^{14}$ Critique 239, avril 1967
} 
littérature française: Miracle de la Rose de Jean Genet et Les Misérables de Victor Hugo. Commençons par Miracle de la Rose :

A dix-sept ans, les Tourelles n'étaient pour moi qu'un nom que j'avais découvert à la fin du livre de Jean Genet, Miracle de la Rose. Il y indiquait les lieux où il avait écrit ce livre : LA SANTÉ, PRISON DES TOURELLES 1943. Lui aussi avait été enfermé là, en qualité de droit commun, peu de temps après le départ de Dora Bruder, et ils auraient pu se croiser. [...] De ce livre, je connaissais des phrases par cœur. L'une d'entre elles me revient en mémoire : «Cet enfant m'apprenait que le vrai fond de l'argot parisien, c'est la tendresse attristée. » Cette phrase m'évoque si bien Dora Bruder que j'ai le sentiment de l'avoir connue. On avait imposé des étoiles jaunes à des enfants aux noms polonais, russes, roumains, et qui étaient si parisiens qu'ils se confondaient avec les façades des immeubles, les trottoirs, les infinies nuances de gris qui n'existent qu'à Paris. Comme Dora Bruder, il parlaient tous avec l'accent de Paris, en employant des mots d'argot dont Jean Genet avait senti la tendresse attristée ${ }^{15}$.

La tendresse attristée sentie par Genet à propos du parler des enfants juifs à Paris sous l'Occupation, voilà ce qui crée pour Modiano un dialogue entre son roman en train de naître et celui de Genet, rédigé sous cette Occupation. C'est une intertextualité chronotopique, qui est liée au temps et à l’espace d'une façon on ne peut plus nette et précise : « la Santé, prison de Tourelles $1943 »$.

Le dialogue avec le roman de Victor Hugo se fait d'une autre manière, toujours chronotopique mais en outre plus proche de l'effet de voyance, si important pour Modiano. D'abord, le narrateur précise en s'y situant, le temps de la narration :

J'écris ces pages en novembre 1996. Les journées sont souvent pluvieuses. Demain nous entrerons dans le mois de décembre et cinquante-cinq ans auront passé depuis la fugue de Dora. [...] J'ai l'impression d'être tout seul à faire le lien entre le Paris de ce temps-là et celui d'aujourd'hui, le seul à me souvenir de tous ces détails. Par moment, le lien s'amenuise et risque de se rompre, d'autres soirs la ville d'hier m'apparaît en reflet furtifs derrière elle d'aujourd'hui.

J'ai relu les livres cinquième et sixième des Misérables ${ }^{16}$.

A ce moment-là, dans le texte romanesque, le narrateur donne une description de la traversée nocturne de Paris faite par les personnages fictifs hugoliens, Cosette et Jean Valjean, traqués par un autre personnage fictif, Javert. Sur un plan de Paris on peut facilement suivre une partie de cette traversée, depuis le quartier de la barrière Saint-Jacques, le long du Jardin des Plantes et à travers le Pont d'Austerlitz. Et c'est alors qu'arrive le suivant (présenté par le narrateur de Dora Bruder) :

Á peine Jean Valjean a-t-il mis le pied sur la rive droite qu'il croit que des ombres s'engagent sur le pont. La seule manière de leur échapper - pense-t-il - c'est de suivre la petite rue du chemin Vert-Saint-Antoine.

Et soudain, on éprouve une sensation de vertige, comme si Cosette et Jean Valjean, pour échapper à Javert et à ses policiers, basculent dans le vide : jusque-là, ils traversaient les

\footnotetext{
${ }^{15}$ Dora Bruder, pp. 138-139.

${ }^{16}$ Ibid, pp. 50-51.
} 
vraies rues du Paris réel, et brusquement ils sont projetés dans le quartier d'un Paris imaginaire que Victor Hugo nomme le Petit Picpus ${ }^{17}$.

Le narrateur compare cette sensation de vertige à une sensation d'étrangeté qu'on éprouve lorsqu'on marche en rêve dans un quartier inconnu, et au réveil, on réalise peu à peu que les rues de ce quartier étaient décalquées sur des rues familières pour vous le jour. Et voilà que l'incroyable « coïncidence » se présente : le jardin d'un couvent imaginaire où Cosette et Jean Valjean vont se cacher est situé, par le romancier Victor Hugo, exactement au 62 de la rue du Petit-Picpus, la même adresse que celle du pensionnat de Cœur-de-Marie, donc celui où vivait Dora Bruder et d'où elle allait faire une fugue « un soir de décembre au cours duquel la neige était peut-être tombée sur Paris ${ }^{18}$. »

Modiano cite alors ce que dit Hugo à propos de cette adresse fictive de la rue du PetitPicpus : «A l'époque où se passe cette histoire - écrit Hugo - un pensionnat était joint au couvent $^{19}$. »Victor Hugo fait ensuite une description minutieuse de ces lieux pour terminer ce paragraphe des Misérables par un commentaire métalittéraire très intéressant pour le lecteur de Dora Bruder, qui vient d'apprendre le lien intertextuel entre les romans Dora Bruder et Les Misérables notamment au niveau topique, concernant le lieu à la fois imaginaire, chez Hugo, et réel, chez Modiano, le couvent à l'adresse 62 de la rue du Petit-Picpus. Le narrateur modianien termine le paragraphe de Dora Bruder en citant le commentaire suivant du narrateur hugolien des Misérables: "'Nous n'avons pu passer devant cette maison extraordinaire, inconnue, obscure, sans y entrer et sans faire entrer les esprits qui nous accompagnent et qui nous écoutent raconter, pour l'utilité de quelques-uns peut-être, l'histoire mélancolique de Jean-Valjean' ${ }^{20}$. »

Le narrateur de Dora Bruder enchaine en parlant du « don de voyance chez les romanciers ", tel que nous l'avons vu dans la citation liminaire du présent article. Pour Modiano, ce don « fait partie du métier », et en effet, nous venons de voir un bel exemple de ce phénomène dans Les Misérables, où le romancier Hugo imagine un couvent fictionnel à l'adresse même où se trouve le pensionnat du Saint-Cœur-de-Marie, d'où Dora Bruder fera sa fugue en décembre 1942. Coïncidence ou voyance ? Pour encore revenir à notre citation liminaire, ce don, qui à croire Modiano fait partie du métier, « peut sans doute provoquer à la longue de brèves intuitions 'concernant des événements passés ou futurs', comme l'écrit le dictionnaire Larousse à la rubrique 'Voyance' ». Et en effet, des exemples se trouvent aussi bien chez Hugo que chez Modiano.

Concernant ce dernier, passons maintenant à la fin du roman Dora Bruder. Nous allons voir comment le phénomène de voyance joue dans la conscience du romancier, cette fois dans sa conscience éveillée et lucide, donc pas «inconsciemment» comme dans la citation ci-dessus des pages 53-54 (voir note 11). Regardons donc l'avant-dernier paragraphe du roman :

Le samedi 19 septembre, le lendemain du départ de Dora et son père, les autorités d'occupation imposèrent un couvre-feu en représailles à un attentat qui avait été commis au cinéma Rex. Personne n'avait le droit de sortir, de trois heures de l'après-midi

\footnotetext{
${ }^{17}$ Ibid, p. 51

${ }^{18}$ Ibid, p. 54

${ }^{19}$ Ibid, p. 52.

${ }^{20}$ Loc.cit.
} 
jusqu'au lendemain matin. La ville était déserte, comme pour marquer l'absence de Dora.

Depuis, le Paris où j'ai tenté de retrouver sa trace est demeuré aussi désert et silencieux que ce jour-là. Je marche à travers les rues vides. Pour moi elles le restent, même le soir, à l'heure des embouteillages, quand les gens se pressent vers le bouches du métro. Je ne peux pas m'empêcher de penser à elle et de sentir un écho de sa présence dans certains quartiers $^{21}$.

«Un écho de sa présence », c'est donc plutôt un écho de l'absence de Dora, pour rester dans le chronotope créé par Modiano, un croisement d'un temps et d'un lieu avec la conscience du narrateur, la conscience d'une présence absente, ou du présence manquant d'un personnage de roman. Ce personnage romanesque est à la fois fictionnel et historique, sans aucun doute fictionnel tel qu'il est décrit par Modiano dans le passage cité de la fin de son roman, mais en même temps historique en tant que victime véritable et documentée d'une destinée collective et atroce, appelée la Shoah ou l'Holocauste. L'approche chronotopique nous aide à mieux comprendre la partie soulignée de la dernière citation : si pour le narrateur, les rues restent vides, même le soir, à l'heure des embouteillages, quand les gens se pressent vers le bouches du métro, c'est précisément parce qu'il ne peut pas s'empêcher de penser à elle et de sentir un écho de sa présence dans certains quartiers.

La vie et la mort d'une jeune fille nommée Dora Bruder seraient oubliées pour toujours si le roman de Modiano n'avait pas été écrit. L'emploi du chronotope renforce à mon avis à la fois l'effet de commémoration de la Shoah et de présence individuelle, quoique fictionnelle, dans cette destinée collective. Le chronotope nous fait vivre la mémoire de Dora dans les rues de Paris sous l'Occupation d'une manière difficile sinon impossible à évoquer dans un récit historique sans cette « voyance » narrative qui caractérise la prose romanesque modianienne dans le passage cité. Voilà « la corrélation essentielle des rapports spatiotemporels, telle qu'elle a été assimilée par la littérature », telle formulée par Bahktine dans sa définition du chronotope dans notre citation donnée plus haut.

Nous avons vu comment ce chronotope peut fonctionner à l'intérieur d'une relation intratextuelle entre deux romans de Modiano, dans les réflexions métalittéraires sur le rapport entre les romans Voyage de noces et Dora Bruder, données en plein milieu d'un d'eux, Dora Bruder. De plus, nous avons vu le fonctionnement du chronotope à l'intérieur d'une relation intertextuelle entre deux romans d'auteurs différents, notamment entre Les Misérables et Dora Bruder. Dans ce dernier exemple, nous avons pu remarquer comment la voyance des deux romanciers crée des espaces ou lieux fictifs dans un temps imaginaire, comme dans le chronotope lié à l'adresse du pensionnat d'où Dora Bruder fit sa fugue dans le temps réel de décembre 1942 et où Cosette se cachait avec Jean Valjean environ un siècle auparavant dans un lieu et un espace de temps fictifs, imaginés par Victor Hugo.

Une telle "corrélation essentielle des rapports spatio-temporels," (pour parler comme Bahktine) liée aux "efforts d'imagination, nécessaires à ce métier," (pour parler comme Modiano), une telle corrélation chronotopique ouvrent, selon moi, de nouvelles portes vers une compréhension approfondie de l'œuvre romanesque en question. Elle nous présente la situation vécue par une jeune juive nommé Dora Bruder au travers d'une situation non vécue mais imaginée par un romancier nommé Patrick Modiano, né après la Shoah mais profondément marqué par elle. Ce chronotope, cette corrélation spatio-temporel, éloigne la

\footnotetext{
${ }^{21}$ Dora Bruder, p.144. C'est moi qui souligne.
} 
description littéraire de la réalité, fût-elle historique (donc appartenant au temps de l'histoire ou temps raconté) ou présente (donc appartenant au temps du récit ou temps de la narration), tout en lui donnant une dimension intertextuelle, ainsi enrichissant la narration de la fugue de Dora. Ce chronotope intertextuel n'appartient ni au temps de l'histoire ni au temps de la narration. Elle appartient au temps et lieu de la voyance, de « cette tension, cette gymnastique cérébrale [qui] peut sans doute provoquer à la longue de brèves intuitions 'concernant des événements passés ou futurs' ${ }^{22}$. Espérons que les événements de la Shoah relatés dans Dora Bruder concernent uniquement des événements passés et non futurs.

\section{Bibliographie}

Bakhtine, Mikhaïl M. 1978. Esthétique et théorie du roman. Paris : Gallimard.

Bem, Jeanne 2000. «Dora Bruder ou la biographie déplacée de Modiano ». Cahiers de l'Association international des études française 52, pp. 221-232.

Genette, Gérard 1972. «Discours du récit: essai de méthode » In Figures III, Paris: Seuil, pp. 165-267.

Greve, A. \& Gaasland, R. 2004. « $\AA$ snakke med hverandre. Om fagfellesskap, begrepsforvaltning og personlig engasjement i litteraturvitenskapen ». Norsk Litteraturvitenskapelig Tidsskrift 1/2004, pp. 32-44.

Holm, Helge V. 2017. « Chronotopes in Patrick Modiano's Fictional Writing of History ». In Dyvik Cardona, Koppen \& Lunde (eds): Remaining Relevant - Modern Laguage Studies Today. BeLLS vol. 7 (2017), pp. 103-111.

Hilsum, Mireille 2012. «Serge Klarsfeld/Patrick Modiano: enjeux d'une occultation». L'Herne 1/2012, pp. 187-191.

Kristeva, Julia 1967. «Bakhtine, le mot, le dialogue et le roman ». Critique 239 (avril 1967), pp. $438-465$.

Modiano, Patrick 1990. Voyage de noces. Paris : Gallimard.

Modiano, Patrick 1997. Dora Bruder. Paris : Gallimard. Edition Folio 1999.

\footnotetext{
${ }^{22}$ Voir notre citation liminaire.
} 